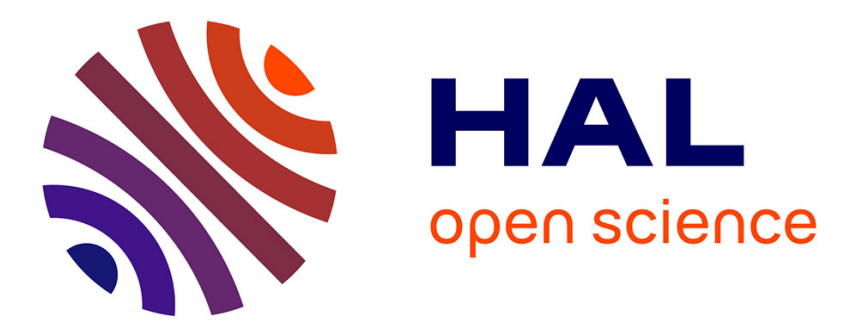

\title{
Orientational anisotropy in simulated vapor-deposited molecular glasses
}

\author{
Ivan Lyubimov, Lucas Antony, Diane M. Walters, David Rodney, M. D. \\ Ediger, Juan J. de Pablo
}

\section{- To cite this version:}

Ivan Lyubimov, Lucas Antony, Diane M. Walters, David Rodney, M. D. Ediger, et al.. Orientational anisotropy in simulated vapor-deposited molecular glasses. Journal of Chemical Physics, 2015, 143 (9), pp.8. 10.1063/1.4928523 . hal-01298375

\section{HAL Id: hal-01298375 \\ https://hal.science/hal-01298375}

Submitted on 4 Feb 2021

HAL is a multi-disciplinary open access archive for the deposit and dissemination of scientific research documents, whether they are published or not. The documents may come from teaching and research institutions in France or abroad, or from public or private research centers.
L'archive ouverte pluridisciplinaire HAL, est destinée au dépôt et à la diffusion de documents scientifiques de niveau recherche, publiés ou non, émanant des établissements d'enseignement et de recherche français ou étrangers, des laboratoires publics ou privés. 


\section{AlP | $\begin{aligned} & \text { The Journal of } \\ & \text { Chemical Physics }\end{aligned}$}

\section{Orientational anisotropy in simulated vapor-deposited molecular glasses}

Ivan Lyubimov, Lucas Antony, Diane M. Walters, David Rodney, M. D. Ediger, and Juan J. de Pablo

Citation: The Journal of Chemical Physics 143, 094502 (2015); doi: 10.1063/1.4928523

View online: http://dx.doi.org/10.1063/1.4928523

View Table of Contents: http://scitation.aip.org/content/aip/journal/jcp/143/9?ver=pdfcov

Published by the AIP Publishing

\section{Articles you may be interested in}

Vapor-deposited non-crystalline phase vs ordinary glasses and supercooled liquids: Subtle thermodynamic and kinetic differences

J. Chem. Phys. 142, 164510 (2015); 10.1063/1.4918745

How much time is needed to form a kinetically stable glass? AC calorimetric study of vapor-deposited glasses of ethylcyclohexane

J. Chem. Phys. 142, 054506 (2015); 10.1063/1.4906806

Vapor-deposited $\alpha, \alpha, \beta$-tris-naphthylbenzene glasses with low heat capacity and high kinetic stability

J. Chem. Phys. 137, 154502 (2012); 10.1063/1.4758807

Dynamics of glass-forming liquids. XV. Dynamical features of molecular liquids that form ultra-stable glasses by vapor deposition

J. Chem. Phys. 135, 124515 (2011); 10.1063/1.3643332

Observation of low heat capacities for vapor-deposited glasses of indomethacin as determined by AC nanocalorimetry

J. Chem. Phys. 133, 014702 (2010); 10.1063/1.3442416

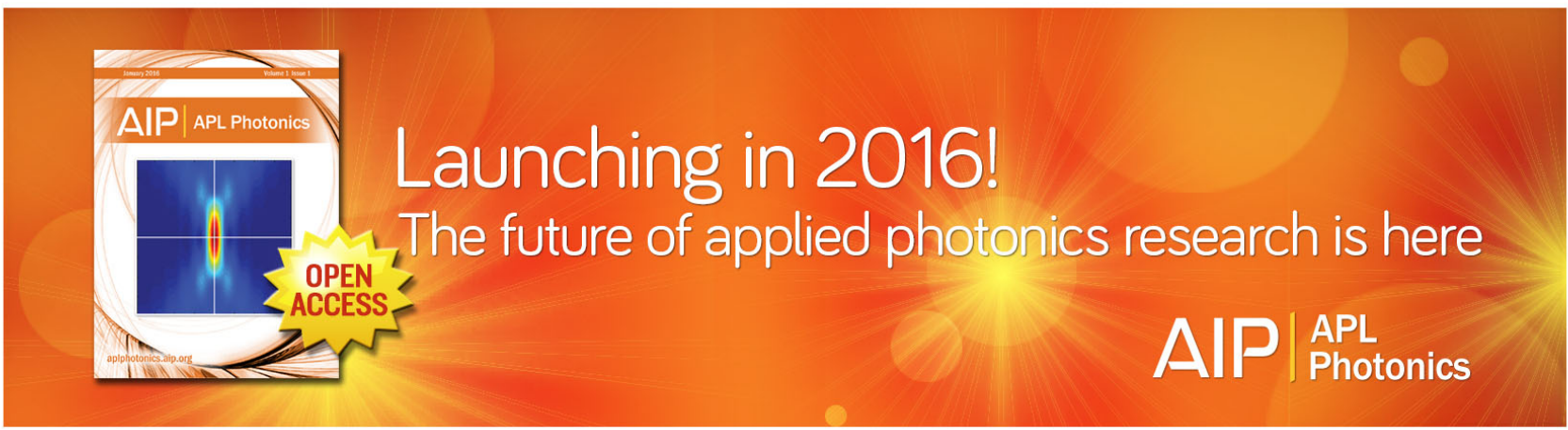




\title{
Orientational anisotropy in simulated vapor-deposited molecular glasses
}

\author{
Ivan Lyubimov, ${ }^{1}$ Lucas Antony, ${ }^{1}$ Diane M. Walters, ${ }^{2}$ David Rodney, ${ }^{3,4}$ M. D. Ediger, ${ }^{2}$ \\ and Juan J. de Pablo ${ }^{1,5, a)}$ \\ ${ }_{1}^{1}$ Institute for Molecular Engineering, University of Chicago, Chicago, Illinois 60637, USA \\ ${ }^{2}$ Department of Chemistry, University of Wisconsin-Madison, Madison, Wisconsin 53706, USA \\ ${ }^{3}$ Science et Ingénierie des Matériaux et Procédés, Grenoble INP, CNRS/UJF, \\ 38402 Saint Martin d'Hères, France \\ ${ }^{4}$ Institut Lumière Matière, Universitè Claude Bernard Lyon, CNRS, 69622 Villeurbanne, France \\ ${ }^{5}$ Argonne National Laboratory, Argonne, Illinois 60439, USA
}

(Received 27 April 2015; accepted 28 July 2015; published online 1 September 2015)

\begin{abstract}
Enhanced kinetic stability of vapor-deposited glasses has been established for a variety of glass organic formers. Several recent reports indicate that vapor-deposited glasses can be orientationally anisotropic. In this work, we present results of extensive molecular simulations that mimic a number of features of the experimental vapor deposition process. The simulations are performed on a generic coarse-grained model and an all-atom representation of $\mathrm{N}, \mathrm{N}^{\prime}$-bis(3-methylphenyl)-N, $\mathrm{N}^{\prime}$ diphenylbenzidine (TPD), a small organic molecule whose vapor-deposited glasses exhibit considerable orientational anisotropy. The coarse-grained model adopted here is found to reproduce several key aspects reported in experiments. In particular, the molecular orientation of vapor-deposited glasses is observed to depend on substrate temperature during deposition. For a fixed deposition rate, the molecular orientation in the glasses changes from isotropic, at the glass transition temperature, $T_{g}$, to slightly normal to the substrate at temperatures just below $T_{g}$. Well below $T_{g}$, molecular orientation becomes predominantly parallel to the substrate. The all-atom model is used to confirm some of the equilibrium structural features of TPD interfaces that arise above the glass transition temperature. We discuss a mechanism based on distinct orientations observed at equilibrium near the surface of the film, which get trapped within the film during the non-equilibrium process of vapor deposition. (C) 2015 AIP Publishing LLC. [http://dx.doi.org/10.1063/1.4928523]
\end{abstract}

\section{INTRODUCTION}

Glasses are amorphous solids that can be formed by a variety of different routes. ${ }^{1}$ The most common route employed in systematic studies of glasses relies on cooling a liquid below its glass transition temperature, $T_{g}$, in a manner that avoids crystallization. ${ }^{2}$

It has recently been established both experimentally and in computer simulations that ultra-stable glasses can be prepared by physical vapor deposition (PVD). ${ }^{3-18}$ When the substrate temperature is held somewhat below $T_{g}$, vapordeposited glasses can exhibit properties that are superior to those of ordinary liquid-cooled glasses. Enhanced kinetic stability and lower enthalpy, ${ }^{5,10,18-21}$ higher density, ${ }^{4,22}$ and higher mechanical moduli ${ }^{8,23,24}$ have now been demonstrated for many different organic glasses prepared by PVD. Very recent work has established that highly stable PVD glasses also have striking low temperature properties which suggest a suppression of two level systems. ${ }^{25}$ Enhanced stability has also been reported for metallic vapor-deposited glasses. ${ }^{13,14}$ Stable glasses have been prepared from a wide range of organic molecules, including indomethacin (IMC), 3,20,26 toluene and ethylbenzene, ${ }^{4,27,28}$ an assortment of alkylbenzenes, ${ }^{29}$ several

\footnotetext{
a) Author to whom correspondence should be addressed. Electronic mail: depablo@uchicago.edu
}

tris-naphthylbenzene (TNB) isomers, ${ }^{30}$ and several organic semiconductors. ${ }^{24,31,32}$

Molecular simulations have also been used to mimic the physical vapor deposition process of a disaccharide,${ }^{33}$ metallic glasses, ${ }^{15,16}$ and short-chain polymeric glasses. ${ }^{17}$ Consistent with experimental observations, simulated PVD-glasses are more kinetically stable than their ordinary liquid-cooled counterparts.

The enhanced molecular mobility at the free interface of PVD glasses is thought to be responsible for their stability. ${ }^{34}$ Indeed, extensive experimental and simulation studies, ${ }^{3,35-38}$ including surface grating decay measurements, ${ }^{39-42}$ have established the enhanced surface mobility of glassy films. Those findings are also qualitatively consistent with the random first order transition (RFOT) theory ${ }^{43}$ and the coupling model of Capaccioli et al. ${ }^{44}$ Enhanced mobility allows molecules near the free surface to sample different packing arrangements during vapor deposition and to find low energy configurations. ${ }^{3}$

There have been only a small number of structural studies of PVD glasses. Studies of PVD glasses of IMC reported an additional anisotropic peak relative to the ordinary glass peak in wide-angle X-ray scattering. ${ }^{25,45,46}$ More recent measurements on different isomers of TNB have also revealed structural anisotropy, which to some extent varies depending on the isomer. ${ }^{47}$ Grazing-incidence $\mathrm{x}$-ray scattering measurements 
on N,N'-bis(3-methylphenyl)-N, $\mathrm{N}^{\prime}$-diphenylbenzidine (TPD) indicate anisotropy in nearest-neighbor packing that depends upon substrate temperature during deposition. ${ }^{48}$ Based on these last two studies, it has been suggested that anisotropic packing is not a necessary condition for high kinetic stability, but rather that these two properties are independent features of the vapor deposition process. A recent analysis of model PVD polymer glasses reinforces that view. ${ }^{17}$

Molecular orientational anisotropy and birefringence have also been measured by Dalal and Ediger in PVD glasses of IMC $^{49}$ and $\alpha, \alpha, \beta$-TNB glasses. ${ }^{22}$ Recent work by Yokoyama et al. has provided new insights into orientational anisotropy and its relation to device performance. Specifically, by examining PVD glasses of several semiconductor organic materials, these authors reported that molecular orientation can be manipulated by molecular shape and deposition conditions. ${ }^{50-52}$ It has been demonstrated that a change in anisotropic orientation can lead to better charge carrier transport properties than those observed in isotropic materials..$^{51,53,54}$ These findings offer potential for improvement of organic light emitting diodes (OLEDs) and other organic electronic and optoelectronic devices. ${ }^{55}$ To realize that potential, however, it is important to understand what governs orientational anisotropy, to what extent the orientational anisotropy can be controlled, and which types of molecules are most susceptible to exhibit pronounced effects. Several factors controlling orientation anisotropy in organic vapor deposited films have been proposed, including molecular shape, ${ }^{50,52}$ surface roughness, ${ }^{51}$ substrate temperature, ${ }^{31,49,52}$ and the glass transition temperature of the bulk material. ${ }^{31,56,57}$

In this work, we address those issues by investigating the mechanisms leading to orientational anisotropy in PVD glasses of a coarse-grained model of TPD. This molecule has a slightly elongated shape. A recent study of TPD and two other compounds with similar molecular structures systematically investigated the influence of substrate temperature on molecular orientation. ${ }^{31}$ We adopt a purposely simple model that only captures the molecule's shape, and we show that such a model is sufficient to describe a number of non-trivial features observed in experiments. The predictions of that model are validated by performing a limited set of simulations using a detailed atomistic representation of TPD. Due to computational limitations, however, the allatom simulations are limited to equilibrium calculations well above the glass-transition temperature. All of our simulation results are consistent with the view that, during deposition, molecules at the free surface strive to attain molecular orientations consistent with the surface of the equilibrium liquid.

The manuscript is organized as follows. In Section II, we introduce the coarse-grained TPD model and discuss the details of our simulations of PVD films. We present the details of our all-atom simulations of TPD films at equilibrium. In Section III, we present the main results of our calculations and a discussion of the relation between molecular orientation in PVD glasses and interfacial properties of the equilibrium liquid. Section IV provides a summary of our findings. A partial account of this work, particularly the experimental aspects, was published recently. ${ }^{31}$

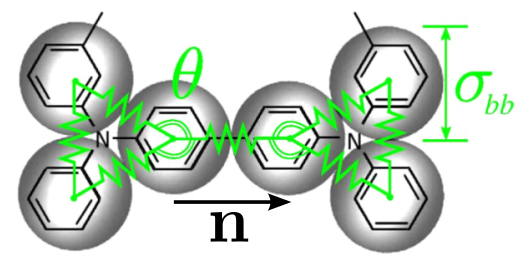

FIG. 1. Schematic representation of coarse-grained model for TPD molecule.

\section{METHODS}

The coarse-grained TPD model considered here consists of six spherical particles, representing the benzene rings of the actual molecule (Figure 1). Each sphere interacts through a Lennard-Jones (LJ) potential with parameters $\sigma_{b b}=1.0$, $\epsilon_{b b}=1.0$. The cutoff distance for the potential is $r_{c}=2.5$ with a smooth decay starting at a 2.4 separation. To maintain the intra-molecular structure, the six LJ particles of a molecule are connected by seven stiff bonds $\left(l_{b}=1.0, k_{b}=1000\right)$. Angle potentials are applied to four groups of three particles that include two interior beads and one of the exterior beads $\theta$ $\left.=150^{\circ}, k_{\text {angle }}=1000\right)$. The orientation of the molecule is characterized by a unit vector $\mathbf{n}$ connecting the two interior beads. No additional restrictions are applied to the relative rotation of the two halves of the molecule about the longitudinal axis, $\mathbf{n}$.

The simulation box size used here was $20 \sigma_{b b}$ by $20 \sigma_{b b}$ in the plane of the substrate, and at least $10 \sigma_{b b}$ larger than the deposited film thickness normal to the substrate (Figure 2). Periodic boundary conditions were applied in directions parallel to the substrate ( $x-y$ plane), which was generated from 1000 randomly placed smaller LJ particles. The LJ potential

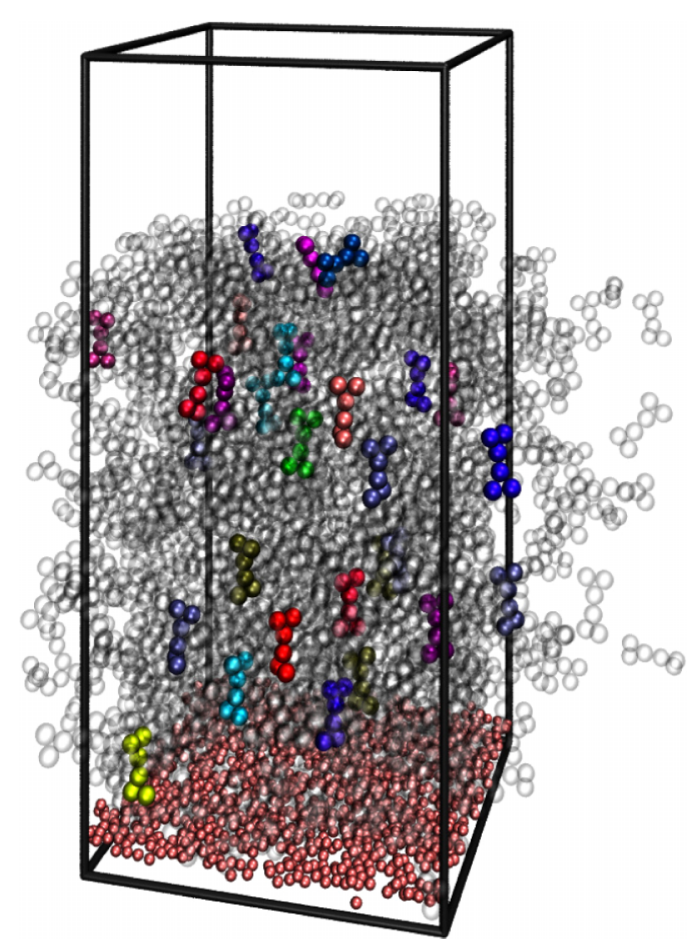

FIG. 2. A snapshot from the video clip of deposition simulation. The molecules with vertical final orientation are highlighted in colors. The substrate particles are shown in red. (Multimedia view) [URL: http://dx.doi.org/ $10.1063 / 1.4928523 .1]$ 
parameters for the substrate were chosen in such a way as to minimize their effect on the deposited molecules and prevent undesirable ordering. ${ }^{58}$ The parameters for the interaction with other substrate atoms are $\sigma_{s s}=0.6, \epsilon_{s s}=0.1$, and with beads representing benzene rings, they are $\sigma_{s b}=1.0$ and $\sigma_{s b}=1.0$, with a cutoff distance of $2.5 \sigma_{\alpha \beta}$, where $\alpha, \beta \in s, b$. All substrate atoms were fixed in their random initial position with harmonic springs with force constant $k=1000$.

The simulated vapor deposition process was analogous to that reported earlier for simple binary glasses and polymeric glasses. ${ }^{15,17}$ Iterative cycles of deposition and annealing were repeated until a film with a thickness of approximately $35 \sigma_{b b}$ was grown. Specifically, each cycle consisted of (1) introduction of 4 new molecules at a time in the proximity of the film surface and with random orientation, (2) equilibration of newly introduced molecules at high temperature $(T=1.0)$, (3) cooling of these molecules to the substrate temperature at a constant rate, and (4) energy minimization of the entire system. The previously deposited molecules and the substrate particles were maintained at a constant temperature throughout the process using a separate thermostat. Different deposition rates were achieved by changing the number of time steps assigned for cooling newly introduced molecules to the substrate temperature during the third stage of each cycle. $7 \times 10^{5}$, $21 \times 10^{5}$, and $2 \times 10^{5}$ time steps were used for normal, slower, and faster deposition rates, respectively. All simulations were performed using the LAMMPS simulation package ${ }^{59}$ in the NVT ensemble with the simulation time step of 0.001. All samples were properly relaxed to diminish the influence of thermostat; after that relaxation, the properties of deposited glasses below $T_{g}$ were not changed during computationally accessible times. Relevant data were collected from the middle region of the films in order to reduce the effects of the substrate and the free surface. The thickness of the middle region was $10 \sigma_{b b}$, and the distance from the region bounds to the substrate or to the free surface exceeded $10 \sigma_{b b}$. All quantities pertaining to coarse-grained simulations are given in Lennard-Jones units.

All-atom simulations of TPD liquid films were performed with the all-atom optimized potential for liquid simulations (AA-OPLS) force field. ${ }^{60,61}$ The substrate consisted of Lennard-Jones particles with characteristic energy and size similar to those of silicon $(\sigma=3 \AA, \epsilon=5.2 \mathrm{~kJ} / \mathrm{mol}$, mass $=60$, and density $=2650 \mathrm{~g} / \mathrm{l}$ ) as used in previous all-atom PVD simulations. ${ }^{33}$ Each substrate atom was restrained to its initial position by a harmonic potential with spring constant $K$ $=10^{4} \mathrm{~kJ} /\left(\mathrm{mol} \mathrm{nm}{ }^{2}\right)$. The volume of the system was held constant with dimensions $8.5 \mathrm{~nm} \times 8.5 \mathrm{~nm} \times 25 \mathrm{~nm}$ and was periodic in all three dimensions. The $x$ and $y$ dimensions are approximately five times the molecular length of TPD $(\approx 1.7 \mathrm{~nm})$. The length of the box in the $z$ direction (perpendicular to the substrate) was sufficiently large to encompass the film with ample amount of vacuum to allow for film expansion while avoiding interaction between the top layer and the periodic image of the substrate. Coulombic forces were calculated using the particle mesh Ewald algorithm ${ }^{62}$ with a force and potential correction applied in the $z$ dimension in order to produce a pseudo-2D summation. Molecular simulations were carried out using the GROMACS 4.6.3 simulation package. ${ }^{63,64}$ All simulations were performed with a time step of 2 fs. During production runs, temperature coupling was achieved using velocity rescaling with a stochastic term. ${ }^{65}$

\section{RESULTS}

Using the simulation scheme described above, we prepared deposited glasses of coarse-grained TPD on substrates controlled at temperatures in the range $0.6 \leq T_{\text {sub }} \leq 0.8$, which encompasses the glass transition temperature, $T_{g}=0.7$, of the model system. The simulated glass transition temperature is defined as the fictive temperature, $T_{g}=T_{f}$, measured during the cooling simulation at the slowest cooling rate (see Figure 5). To characterize molecular orientation anisotropy inside the films, we relied on an orientational order parameter defined through a second Legendre polynomial of the form

$$
S_{z}=\left\langle P_{2}\left(\mathbf{n} \cdot \mathbf{n}_{z}\right)\right\rangle=\frac{3}{2}\left\langle\left(\mathbf{n} \cdot \mathbf{n}_{z}\right)^{2}\right\rangle-\frac{1}{2},
$$

where the dot product is between the unit vector along the molecular axis, $\mathbf{n}$, and the normal to the substrate, $\mathbf{n}_{z}$. The averaging was performed over all molecules in the middle region of the film.

Figure 3 shows profiles for number density, $\rho$, orientation order parameter, $S_{z}$, and Debye-Waller factor, $\left\langle u^{2}\right\rangle$, for glasses deposited at the substrate temperature $T_{\text {sub }}=0.69$. These profiles are given as a function of distance from the substrate. The Debye-Waller factor is defined as the meansquare displacement at a time corresponding to the onset of caging motion. ${ }^{66}$ The "middle" region corresponds to $10<z$ $<20$. Unless otherwise stated, the results presented here correspond to vapor-deposited glasses prepared at the intermediate (normal) deposition rate.

Figure 4 shows the substrate temperature dependence of molecular orientation in the middle of the film for three different deposition rates. The main feature of the substratetemperature dependence shown in Figure 4 is its non-monotonic character. When deposited at $T_{s u b}>0.72>T_{g}$, the orientational order parameter of PVD films is zero, as expected for an equilibrium isotropic liquid. For substrate temperatures just

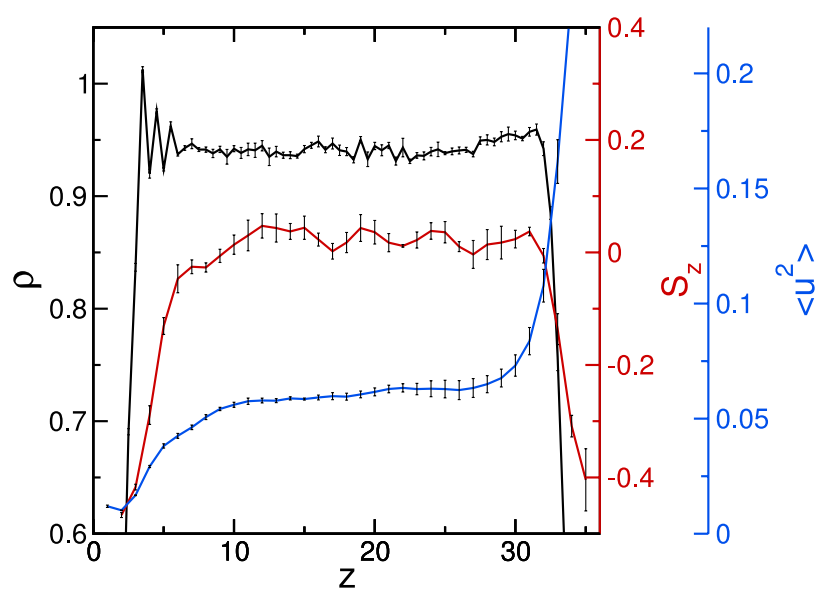

FIG. 3. Structural profiles for vapor deposited glasses prepared at $T_{s u b}$ $=0.69$. The three different curves correspond to three different y-axes: number density (black), orientational order parameter (red), and Debye-Waller factor (blue). 


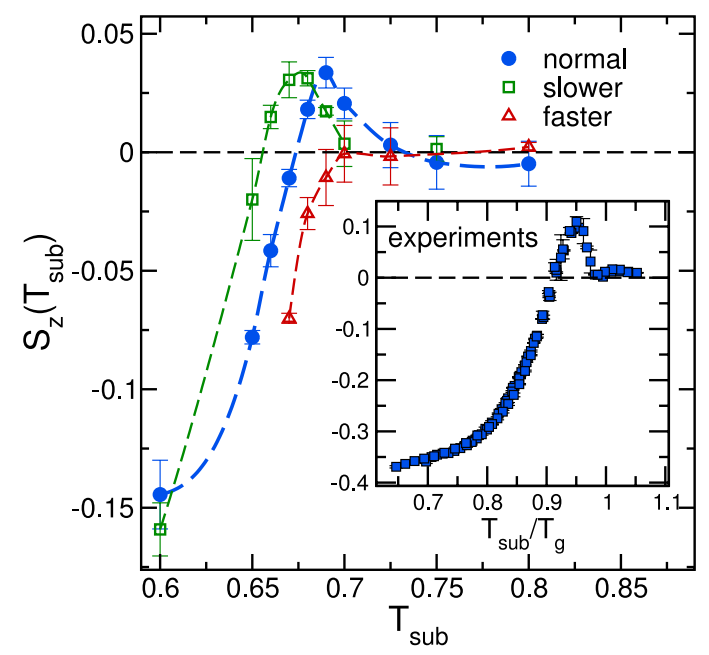

FIG. 4. Orientational order parameter, $S_{z}$, as a function of substrate temperature, $T_{s u b}$, for vapor-deposited glasses prepared at three different deposition rates. The orientation of the deposited material is defined by Eq. (1). The error bars represent the standard deviation of five independent samples. Inset: experimental data showing $S_{z}$ for vapor deposited TPD from Ref. 31 .

below $T_{g}$, molecules exhibit a weak tendency to orient perpendicular to the substrate, as indicated by positive values of $S_{z}$. For even lower $T_{\text {sub }}$, molecules adopt a predominantly parallel orientation to the substrate, with $S_{z}<0$. This behaviour is in qualitative agreement with experimental data of TPD shown in the inset of Figure 4.

In Figure 5, we compare the potential energy of simulated vapor-deposited glasses to that of "ordinary," liquid-cooled glassy thin films. Symbols represent the energy of as-deposited PVD films from Figure 4 prepared at the normal deposition rate. The ordinary glasses were prepared by cooling of the equilibrium liquid at four different cooling rates ranging from $q_{c}=10^{-3}$ to $q_{c}=10^{-6}$. The fictive temperature, $T_{f}$, for each cooling rate was found from the intersection of two slopes representing the equilibrium liquid line and the extrapolated

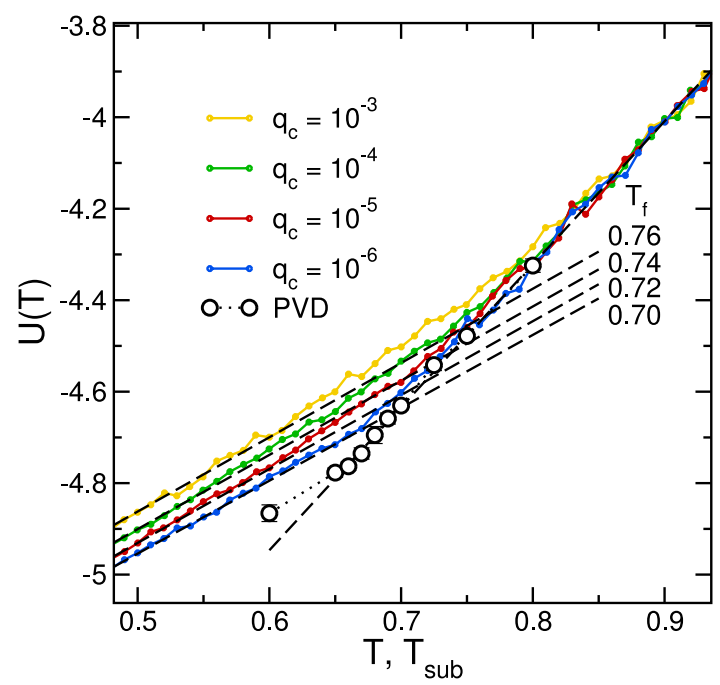

FIG. 5. Potential energy as a function of temperature. Symbols correspond to the energy of as-deposited PVD films prepared at substrate temperatures in the range $0.6 \leq T_{s u b} \leq 0.8$. Colored lines show the potential energy of liquid-cooled films at four different cooling rates ranging from $q_{c}=10^{-3}$ to $q_{c}=10^{-6}$. line from the glassy region. For the system considered here, the cooling-rate dependence of the fictive temperature is linear, and the temperature shift is approximately $\Delta T_{f} \simeq 0.02$ per decade of cooling rate.

In order to assess the kinetic stability of vapor-deposited glasses, we performed temperature ramping simulations analogous to those employed in standard experimental calorimetry procedures, albeit at much faster heating and cooling rates. ${ }^{10,31}$ Figure 6 shows the potential energy as a function of temperature during heating and cooling cycles for the film deposited at the substrate temperature $T_{\text {sub }}=0.69$. That film corresponds to the most vertically oriented molecules. During the first heating ramp, the temperature corresponding to the onset of melting is $T_{o n}=0.82$, which is noticeably higher than that for a reheated film, which is $T_{o n}=0.77$, serving to illustrate the enhanced kinetic stability of PVD films.

The course-grained TPD model used in the simulations discussed above is a good glass former and resists crystallization. It also provides reasonable qualitative agreement with known experimental features of TPD glasses prepared by vapor deposition. Note, however, that the amplitude of the ordering effect observed in the main panel of Figure 4 is about three times weaker than that seen in experiments and shown in the inset. ${ }^{31} \mathrm{We}$ attribute this difference to the simplicity of the model and, in particular, its lack of a dipole moment or polarizability.

The isotropic orientation of films deposited at $T_{s u b} \gtrsim T_{g}$ is expected as in the equilibrium liquid. To understand the origins of the vertical molecular orientation bias that is observed right below $T_{g}$, however, we examine in more detail the deposition simulation performed at $T_{s u b}=0.69$. A snapshot from the late stages of the deposition process is shown on Figure 2. Vertically oriented molecules are highlighted in colors. A video clip of the growth process is available in the Figure 2 (Multimedia view). One can appreciate in the figure and video that most molecular rearrangements and motion occur in the top layer of the film. Diffusion and reorientation cease once the molecules

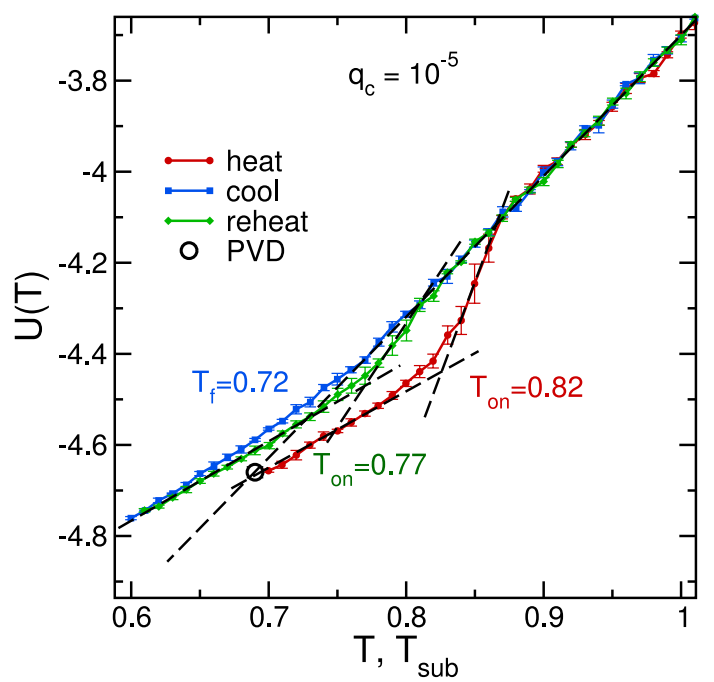

FIG. 6. Potential energy as a function of temperature. The colored lines correspond to heating (red), cooling (blue), and reheating (green) curves starting from a film deposited at $T_{s u b}=0.69$ (symbol). At this substrate temperature, the largest value of $S_{z}$ is observed (most vertical orientation of the molecules). The heating/cooling rate was $q_{c}=10^{-5}$. 


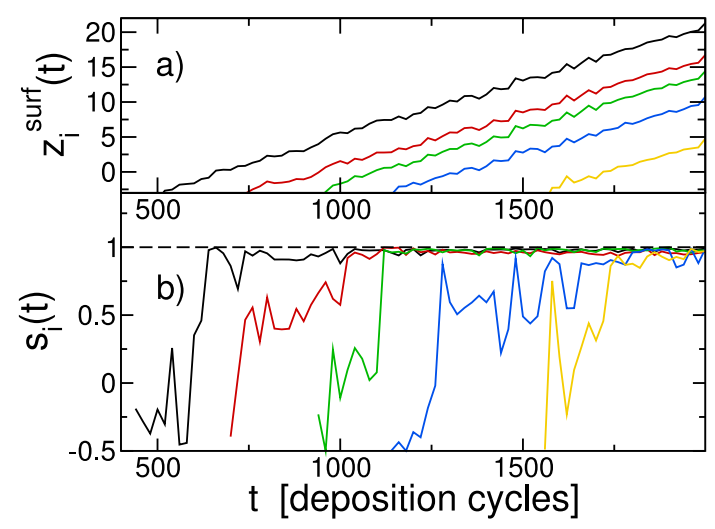

FIG. 7. Individual simulated trajectories of several representative molecules (different colors) during the deposition process: (a) distance from molecule $i$ to the free surface, $z_{i}^{\text {surf }}$, and (b) corresponding individual rotational trajectories, where $s_{i}$ is calculated according to Eq. (2).

lose mobility and are trapped in the "bulk" of the growing film. Newly deposited molecules go through three stages. First, they diffuse on the surface. As the film grows, these molecules are trapped in subsurface regions, where they rotate around one end of the molecule (the "trapped" end). Finally, as deposition and film growth continue, these molecules become completely embedded in the film, and immobilize into their final position and orientation.

Figure 7(a) shows the distance to the free surface, $z_{i}^{\text {surf }}(t)$, as a function of time for several representative molecules. For convenience, the position of the surface is defined as the point where the local density drops to $50 \%$ of the bulk value. The constant slope of $z_{i}^{\text {surf }}(t)$ confirms that no motion occurs along the z-axis, and its value characterizes the deposition rate. To quantify the rotational character of molecular trajectories, we calculate for each molecule the function

$$
s_{i}(t)=P_{2}\left(\mathbf{n}_{i}(t) \cdot \mathbf{n}_{i}^{f}\right),
$$

where $\mathbf{n}_{i}(t)$ is a unit vector along the longitudinal axis of molecule $i$ at time $t$ and $\mathbf{n}_{i}^{f}$ is the orientation of the final immobilized state. In Figure 7(b), we show trajectories for several representative molecules. As $s_{i}(t)$ approaches unity, molecule $i$ approaches its final conformation.

Individual molecular trajectories exhibit considerable variability; the additional analysis on the $y$-axis of Figure 8(a) shows a function $s(t)$ that characterizes the average molecular orientation relative to its final state,

$$
s(t)=\frac{1}{N} \sum_{i=1}^{N} s_{i}\left(t_{i}+t\right),
$$

where $t_{i}$ denotes the moment in time when molecule $i$ was first deposited on the free surface. The averaging was done over $N=500$ molecules in the center section of the film (along $\mathrm{z}$-axis). Due to the linear time dependence of $z_{i}^{\text {surf }}(t)$, it is convenient to plot on the x-axis of Figure 8(a) the average distance from a molecule to the free surface, $\left\langle z_{i}^{\text {surf }}(t)\right\rangle$, defined with an analogous time shift to $s(t)$ for each molecule. The function $s(t)$ is constructed to measure the correlation between the molecular orientation at time $t$ after the moment of deposition and the final orientation in the frozen state. At $t=0$, the deposited molecules are just introduced into the system
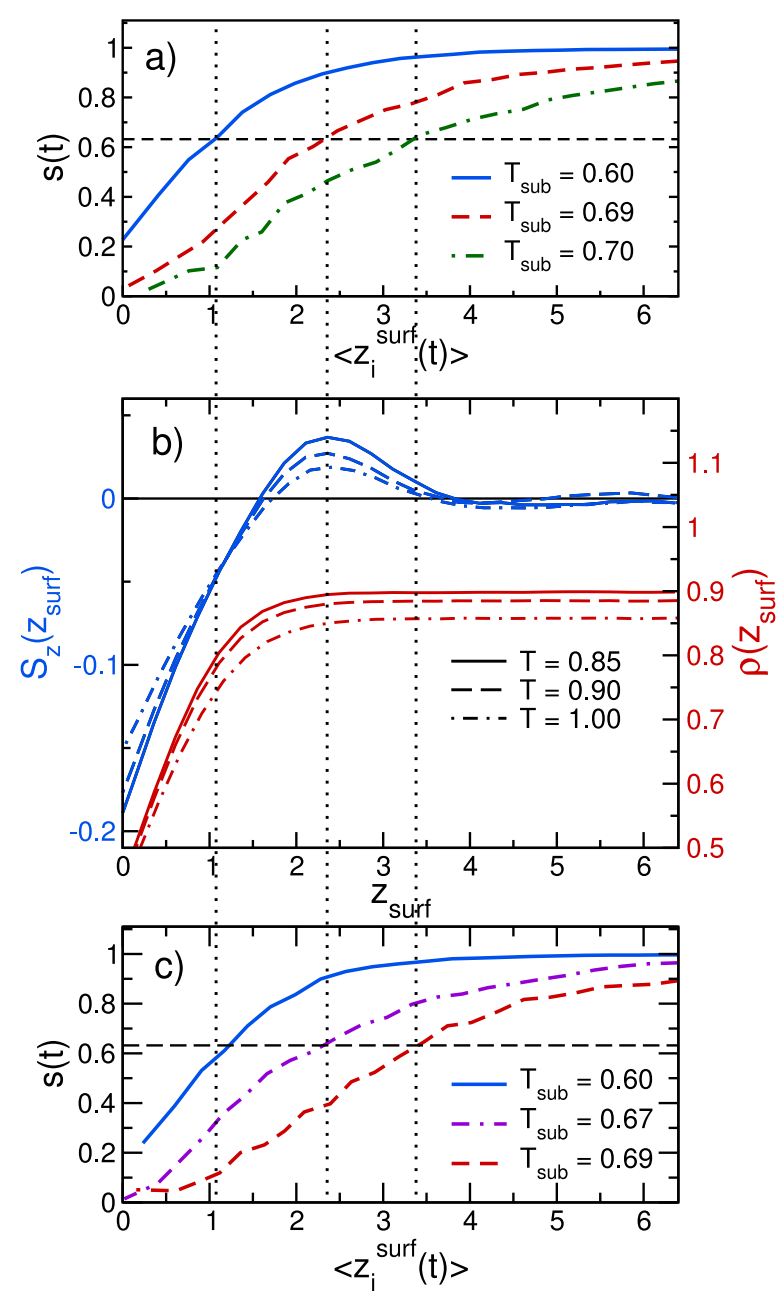

FIG. 8. (a) Correlation function for molecular orientation during deposition at the normal rate, $s(t)$, calculated from Eq. (3). The function is shown for three substrate temperatures: $T_{\text {sub }}=0.60$ (blue), $T_{\text {sub }}=0.69$ (red), and $T_{\text {sub }}=0.70$ (green). (b) Equilibrium liquid profiles of orientational order parameter, $S_{z}$, (blue) and density, $\rho$, (red). The profiles are shown for three temperatures, which are well above the glass transition: $T=0.85$ (solid lines), $T=0.95$ (dashed lines), and $T=1.0$ (dotted-dashed lines). (c) Same functions as in panel (a) calculated for slower deposition rate at $T_{\text {sub }}=0.60$ (blue), $T_{s u b}=0.67$ (violet), and $T_{\text {sub }}=0.69$ (red).

and, at the vacuum interface, $\left\langle z_{i}^{\text {surf }}(t)\right\rangle \simeq 0$. Except for the lowest substrate temperature shown in Figure 8(a), $T_{s}=0.60$, there is no correlation with final molecular orientation at this point. As the deposition process continues, the molecules get buried deeper into the growing film, and the function $s(t)$ smoothly approaches unity, which corresponds to the final immobile state. The lower the substrate temperature, the closer to the surface the immobilization occurs, and, therefore, the shorter the period of time elapsed between the deposition and immobilization.

We define a rotational arrest time, $t_{a}$, through the relation $1-s\left(t_{a}\right)=1 / e$ (horizontal dashed line on Figure 8(a)). We refer to the average distance from the surface corresponding to this moment as the arrest distance, $z_{a}=\left\langle z_{i}^{\text {surf }}\left(t_{a}\right)\right\rangle$. These distances are shown for the three substrate temperatures considered here in Figure 8 by vertical dotted lines.

We now examine the structure of equilibrium liquid films, and focus on the orientational order at the free interface. Figure 8(b) shows the order parameter, $S_{z}$, and the site number 
density, $\rho$, as functions of distance from the free surface, $z_{\text {surf }}$. Results are shown for three different temperatures, well above $T_{g}$. While the density profiles are uniform and decay monotonically at the interface, the orientation order parameter dependence on $z_{\text {surf }}$ exhibits a peak. Right at the free surface, the negative order parameter reflects the tendency for molecules to adopt a parallel orientation. Before reaching an isotropic region inside the film, however, the order parameter becomes positive in the layer $1.5<z_{\text {surf }}<3.5$. In that region, at equilibrium, molecules prefer to exhibit a slight perpendicular orientation.

The height of the slight positive peak of $S_{z}$ in Figure 8(b) increases as temperature decreases towards $T_{g}$; its location remains approximately unchanged with respect to the free surface. The lowest temperature for which results are shown is $T$ $=0.85$. Below that value, it becomes computationally demanding to reach equilibrium. The equilibrium liquid profiles in Figure 8(b) represent a time average over simulations lasting $10^{8}$ time steps (during which more than 5000 configurations were collected). We extrapolate the available data and assume that the height of the positive peak on the $S_{z}$-profile continues to increase as the temperature decreases, without shifting in position.

Figure 8 provides a plausible mechanism for the emergence of molecular orientation in vapor-deposited glasses. For the normal deposition rate, there is reasonable agreement between the arrest distance $z_{a} \simeq 2.3$ for the glass prepared at $T_{\text {sub }}=0.69$ with maximum value of $S_{z}$ and the location of the peak on the $S_{z}$-profile of the equilibrium liquids. At this substrate temperature, molecules with enhanced mobility can penetrate the film down to the depth where the equilibrium liquid configurations exhibit a slightly vertical orientation. As the deposition process continues, that molecular orientation gets locked into the film, giving rise to the anisotropy that is observed inside the films.

At low substrate temperatures, $T_{\text {sub }} \ll T_{g}$, the simulation results shown in Figure 4 indicate that molecules are preferentially orientated horizontally in vapor-deposited glasses of TPD, in good agreement with experimental results. ${ }^{31} \mathrm{We}$ have considered three possible mechanisms to explain this horizontal orientation at low substrate temperatures. (1) Molecules in the top monolayer orient horizontally during deposition in order to equilibrate towards the surface structure of the equilibrium supercooled liquid, which shows horizontal alignment in the topmost layer as shown in Figure 8(b). At low substrate temperatures, there is insufficient mobility for molecules in the second monolayer to equilibrate, and thus the horizontal orientation at of the top monolayer is trapped by further deposition and maintained in the bulk of the film. ${ }^{31}$ (2) At low substrate temperatures, condensing molecules that encounter a flat surface will always prefer to minimize their energy by lying flat on the surface, resulting in a glass in which horizontal deposition is trapped. (3) Molecules deposited on a flat substrate are preferentially oriented horizontally at low temperatures and this initial structure templates further horizontal deposition, similar to what is observed in epitaxial growth. We do not think that this third alternative is realistic given the work of Yokoyama and coworkers..$^{50,67,68}$ They observed that molecular orientation in vapor-deposited films is independent of the orientation of an underlying organic layer, indicating that the substrate does not template molecular orientation over hundreds of molecular layers. At present, we do not have any strong evidence to differentiate between the first two proposed mechanisms.

The equilibrium interfacial behavior shown in Figure 8(b) is central to our explanation of molecular orientation in PVD glasses. The question that arises here is whether that profile is an artifact of our coarse-grained model, or whether it is a feature for the materials considered in this work. To address that question, we begin by noting that past atomistic molecular simulations of ionic liquids have also reported structural anisotropy at the liquid-air interface. ${ }^{69,70}$ For the particular case of TPD, the simulated glass transition temperature for the allatom model was estimated to be $T_{g} \approx 450 \mathrm{~K}$, based on annealing simulations at a rate of $10 \mathrm{~K} / \mathrm{ns}$. Three different simulations containing 783 TPD molecules were conducted at $480 \mathrm{~K}$, $500 \mathrm{~K}$, and $580 \mathrm{~K}$. The simulation at $580 \mathrm{~K}$ totaled $100 \mathrm{~ns}$. Coordinates were saved every $10 \mathrm{ps}$. The simulations at $480 \mathrm{~K}$ and $500 \mathrm{~K}$ were run for $200 \mathrm{~ns}$ each due to the longer relaxation times that are expected closer to $T_{g}$. Figure 9 shows equilibrium liquid profiles of the orientational order parameter, $S_{z}$, and density. Similar to the profiles for the generic model shown on Figure 8(b), the density profile is smooth and does not exhibit any unusual features. The $S_{z}$ profile, however, exhibits a peak in the vicinity of the free surface. The amplitude of this peak is in better quantitative agreement with experiments than that observed with the coarse grained model (inset of Figure 4), serving to further validate the equilibrium structural hypothesis advanced above. The peak grows as temperature decreases, but as the temperature approaches the glass transition it becomes more difficult to equilibrate the simulations. Unfortunately, computational demands prevent us from simulating a vapordeposition process with the all-atom TPD model.

Having put forth a mechanism for the cause of anisotropy in vapor-deposited glasses, one can interpret the results shown in Figure 4 for slower and faster deposition rates. For the slower

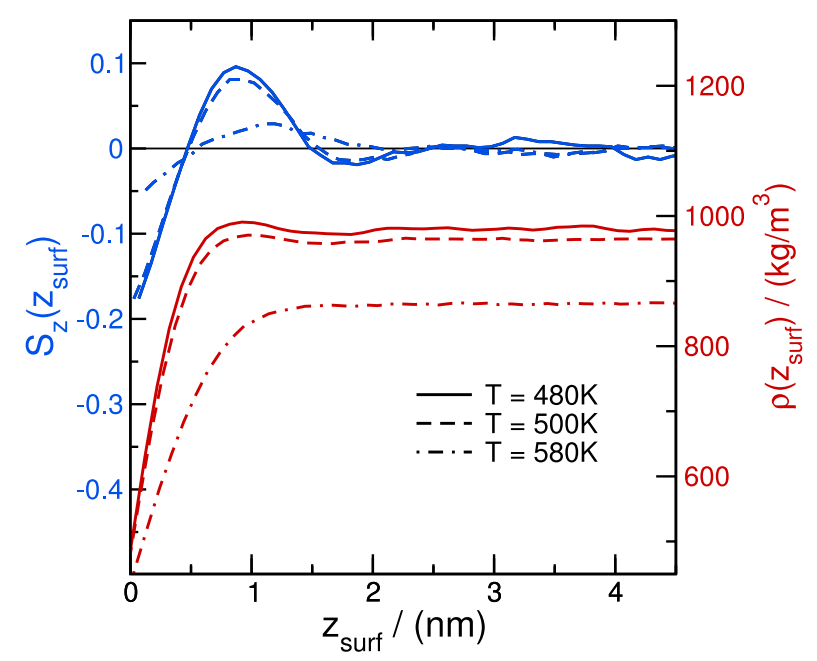

FIG. 9. Equilibrium liquid profiles of orientational order parameter, $S_{z}$, (blue) and density in $\mathrm{kg} / \mathrm{m}^{3}$ (red) obtained from all-atom simulations of TPD. The profiles are given for three temperatures, which are slightly above and well above the simulated glass transition, $T_{g} \approx 450 \mathrm{~K}: T=480 \mathrm{~K}$ (solid lines), $T=500 \mathrm{~K}$ (dashed lines), and $T=580 \mathrm{~K}$ (dotted-dashed lines). 
deposition rate, the $S_{z}$ peak is shifted to the left, towards lower substrate temperatures. This behavior can be understood in terms of the longer time that is available to molecules located in the vicinity of the interface to reach equilibrium. When deposition is slower, at any particular depth from the surface, the molecules spend more time undergoing configurational changes. As a result, the $s(t)$ curves analogous to those shown in Figure 8(a) are stretched along the $\mathrm{x}$-axis for the slower deposition rate as shown in Figure 8(c). Indeed, while the intersection point of the horizontal dashed line and the curve for $T_{\text {sub }}=0.60$ in Figure 8(c) is only marginally shifted to the right in comparison to the corresponding point in Figure 8(a) (solid blue lines), the shift for $T_{\text {sub }}=0.69$ is more significant (dashed red lines). Therefore, the $S_{z}$ peak on Figure 8(b) aligns with the arrest distance for temperature even lower than the $T_{\text {sub }}=0.69$ substrate temperature, $T_{\text {sub }}=0.67$ (dashed-dasheddotted purple line on Figure 8(c)), causing the shift to the left on Figure 4. The points on Figure 4 for the faster deposition are shifted to the higher temperatures instead, and the region with $S_{z}>0$ disappears. At such high deposition rates, however, the simulations and analysis of $s(t)$ functions become less reliable due to approaching computationally applicable limits of the model.

The following analysis of arrest distances further supports the arguments above. Instead of averaging all the rotational trajectories as in function $s(t)$, we now smooth individual trajectories by calculating the time averages over a given interval $\Delta t$ as

$$
\bar{s}_{i}(t)=\frac{1}{\Delta t} \int_{t-\Delta t / 2}^{t+\Delta t / 2} s_{i}\left(t^{\prime}\right) d t^{\prime} .
$$

The value for $\Delta t$ was chosen to be equal to the duration of 40 deposition cycles. The rotational arrest time for each molecule, $t_{i}^{a}$, is defined through the relation $1-\bar{s}_{i}\left(t_{i}^{a}\right)=1 / e$, and it corresponds to a arrest distance $z_{i}^{a}=z_{i}^{\text {surf }}\left(t_{i}^{a}\right)$. Figure 10 shows the distribution of arrest distances for 500 different molecules at the slow deposition rate. Figure 10(b) shows results for $T_{\text {sub }}$ $=0.67$, at which the most pronounced vertical anisotropy is observed. Figure 10(c) shows results for $T_{\text {sub }}=0.60$, at which point the molecules adopt a parallel orientation. The negative values of $z_{i}^{a}$ in Figure 10(a) are attributed to the increased roughness of the free surface of the film deposited at $T_{\text {sub }}$ $=0.60$ compared to that deposited at higher temperatures.

While the distribution $P\left(z_{i}^{a}\right)$ for a low substrate temperature $T_{\text {sub }}=0.60$ appears unimodal, the distributions for higher temperatures develop a tail for large- $z_{i}^{a}$ molecules. This tail becomes more pronounced for $T_{\text {sub }}=0.69$ and corresponds to an increasing fraction of molecules that maintain some rotational mobility inside the film. It reflects a heterogeneity in dynamics occurring at higher temperatures. The distribution $P\left(z_{i}^{a}\right)$ is broadening due to increasing heterogeneity, and the use of a single average value for the arrest distance becomes less sufficient. However, this description is in line with the mechanism discussed above for the data obtained at the normal deposition rate. The average over distribution values for $T_{\text {sub }}=0.60,0.67$, and 0.69 are $\left\langle z_{i}^{a}\right\rangle=1.15,2.57$, and 4.82 correspondingly. The first substrate temperature, $T_{\text {sub }}=0.60$, corresponds to an orientation slightly parallel to the substrate

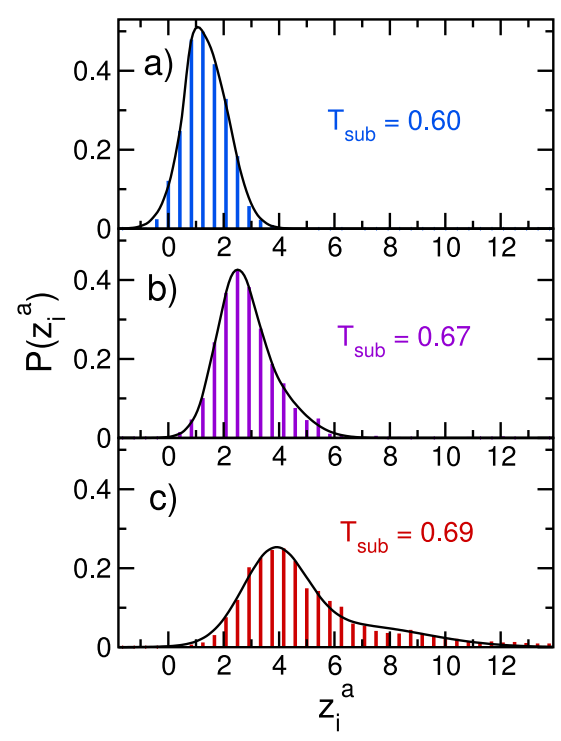

FIG. 10. Distribution function of individual arrest distances, $z_{i}^{a}$, defined in the main text. Distributions shown for the slow deposition rate at three different substrate temperatures: (a) $T_{s u b}=0.60$ (horizontal molecular orientation), (b) $T_{s u b}=0.67$ (most vertical molecular orientation), and (c) $T_{s u b}=0.69$. In all panels, solid lines represent a fit to a sum of two Gaussian functions.

of the PVD glasses, as shown in Figure 4. At the same time, at $z_{\text {surf }}=1.15$, the orientation in the equilibrium liquid is also slightly parallel to the substrate, as shown in Figure 8(b). The second substrate temperature, $T_{\text {sub }}=0.67$, corresponds to the most vertical orientation in the glasses that were deposited at the slower deposition rate (Figure 4). The average value over distribution shown on Figure 10(b) is $\left\langle z_{i}^{a}\right\rangle=2.57$ and aligns well with the peak of positive $S_{z}$-values on the profile from Figure 8(b). For the substrate temperature $T_{\text {sub }}$ $=0.69$, the tendency to orient vertically in glasses deposited at a slower rate is reduced. The equilibrium orientation at the distance corresponding to $\left\langle z_{i}^{a}\right\rangle=4.82$ is isotropic; however, this observation is less reliable due to the growing heterogeneity mentioned above. The average values $\left\langle z_{i}^{a}\right\rangle$ over distributions shown on Figure 10 are also in reasonable agreement with the $z_{a}$ determined from the intersection with the dashed horizontal line in Figure 8(c). Eqs. (3) and (4) represent two different ways of finding the arrest distance. However, both ways are consistent, which supports the provided explanation of orientation in deposited PVD glasses in terms of equilibrium liquid properties at the free interface.

\section{CONCLUSIONS}

A molecular mechanism has been proposed to explain the orientational anisotropy that arises in vapor-deposited glasses of a TPD-like molecule. In particular, the tendency of the molecules to adopt a vertical molecular orientation at a substrate temperature slightly below the bulk glass transition temperature has been explained in terms of the properties of the equilibrium liquid at the free interface. Results of coarse-grained and all-atom molecular simulations provide evidence in support of a driving force that, at equilibrium, orients molecules vertically just below the TPD-vacuum interface. Out of equilibrium and below $T_{g}$, as a film grows in thickness, that local structure 
becomes embedded in the film. When deposition is finished, this results in a positive orientational order parameter. Consistent with experimental observations, our results indicate that vertical anisotropy is most pronounced for substrate temperatures slightly below $T_{g}$. For substrate temperatures well below $T_{g}$, the preferred molecular orientation becomes parallel to the substrate. A new prediction of the model suggests that the peak in vertical anisotropy should be shifted to lower temperatures as deposition rate decreases. We also predict that it should be shifted and eventually disappear as deposition rate increases. We hope that the results of this work will stimulate additional experiments to evaluate such predictions, which could potentially lead to avenues for better control of molecular orientation in PVD glasses.

In conclusion, the simulation results presented in this paper provide a reasonable explanation for anisotropic molecular orientation in vapor-deposited glasses and can be useful in deeper understanding of the vapor deposition process.

\section{ACKNOWLEDGMENTS}

The financial support from the National Science Foundation No. DMR-1234320 is gratefully acknowledged. D.M.W. was supported by the U.S. Department of Energy, Office of Basic Energy Sciences, Division of Materials Sciences and Engineering, Award No. DE-SC0002161.

${ }^{1}$ C. A. Angell, Science 267, 1924 (1995).

${ }^{2}$ P. G. Debenedetti and F. H. Stillinger, Nature 410, 259 (2001).

${ }^{3}$ S. F. Swallen, K. L. Kearns, M. K. Mapes, Y. S. Kim, R. J. McMahon, M. D. Ediger, T. Wu, L. Yu, and S. Satija, Science 315, 353 (2007).

${ }^{4}$ K. Ishii, H. Nakayama, S. Hirabayashi, and R. Moriyama, Chem. Phys. Lett. 459, 109 (2008).

${ }^{5}$ K. L. Kearns, S. F. Swallen, M. D. Ediger, T. Wu, Y. Sun, and L. Yu, J. Phys. Chem. B 112, 4934 (2008).

${ }^{6}$ S. F. Swallen, K. Traynor, R. J. McMahon, M. D. Ediger, and T. E. Mates, Phys. Rev. Lett. 102, 065503 (2009).

${ }^{7}$ S. Leonard and P. Harrowell, J. Chem. Phys. 133, 244502 (2010).

${ }^{8}$ K. L. Kearns, T. Still, G. Fytas, and M. D. Ediger, Adv. Mater. 22, 39 (2010).

${ }^{9}$ S. F. Swallen, K. Windsor, R. J. McMahon, M. D. Ediger, and T. E. Mates, J. Phys. Chem. B 114, 2635 (2010).

${ }^{10}$ S. L. L. M. Ramos, M. Oguni, K. Ishii, and H. Nakayama, J. Phys. Chem. B 115, 14327 (2011).

${ }^{11}$ A. Sepulveda, S. F. Swallen, L. A. Kopff, R. J. McMahon, and M. D. Ediger, J. Chem. Phys. 137, 204508 (2012).

${ }^{12}$ K. R. Whitaker, D. J. Scifo, M. D. Ediger, M. Ahrenberg, and C. Schick, J. Phys. Chem. B 117, 12724 (2013).

${ }^{13}$ H.-B. Yu, Y. Luo, and K. Samwer, Adv. Mater. 25, 5904 (2013).

${ }^{14}$ D. P. B. Aji, A. Hirata, F. Zhu, L. Pan, K. Madhav Reddy, S. Song, Y. Liu, T. Fujita, S. Kohara, and M. Chen, e-print arXiv:1306.1575 [condmat.mtrl-sci] (2013).

${ }^{15}$ S. Singh, M. D. Ediger, and J. J. de Pablo, Nat. Mater. 12, 139 (2013).

${ }^{16}$ I. Lyubimov, M. D. Ediger, and J. J. de Pablo, J. Chem. Phys. 139, 144505 (2013).

${ }^{17}$ P.-H. Lin, I. Lyubimov, L. Yu, M. D. Ediger, and J. J. de Pablo, J. Chem. Phys. 140, 204504 (2014).

${ }^{18}$ D. Bhattacharya and V. Sadtchenko, J. Chem. Phys. 141, 094502 (2014).

${ }^{19}$ S. L. L. M. Ramos, A. K. Chigira, and M. Oguni, J. Phys. Chem. B 119, 4076 (2015).

${ }^{20}$ C. Rodríguez-Tinoco, M. Gonzalez-Silveira, J. Ràfols-Ribé, A. F. Lopeandía, M. T. Clavaguera-Mora, and J. Rodríguez-Viejo, J. Phys. Chem. B 118, 10795 (2014).

${ }^{21}$ A. Wisitsorasak and P. G. Wolynes, Phys. Rev. E 88, 022308 (2013).

${ }^{22}$ S. S. Dalal, A. Sepúlveda, G. K. Pribil, Z. Fakhraai, and M. D. Ediger, J. Chem. Phys. 136, 204501 (2012).

${ }^{23}$ E. A. A. Pogna, C. Rodríguez-Tinoco, G. Cerullo, C. Ferrante, J. RodríguezViejo, and T. Scopigno, Proc. Natl. Acad. Sci. U. S. A. 112, 2331 (2015).

${ }^{24}$ N. Bakken, J. M. Torres, J. Li, and B. D. Vogt, Soft Matter 7, 7269 (2011).
${ }^{25}$ T. Pérez-Castañeda, C. Rodríguez-Tinoco, J. Rodríguez-Viejo, and M. A. Ramos, Proc. Natl. Acad. Sci. U. S. A. 111, 11275 (2014).

${ }^{26} \mathrm{~L}$. Zhu and L. Yu, Chem. Phys. Lett. 499, 62 (2010).

${ }^{27}$ E. León-Gutierrez, G. Garcia, M. Clavaguera-Mora, and J. Rodríguez-Viejo, Thermochim. Acta 492, 51 (2009).

${ }^{28}$ Z. Chen and R. Richert, J. Chem. Phys. 135, 124515 (2011).

${ }^{29}$ K. Ishii, H. Nakayama, R. Moriyama, and Y. Yokoyama, Bull. Chem. Soc. Jpn. 82, 1240 (2009).

${ }^{30}$ K. Dawson, L. Zhu, L. A. Kopff, R. J. McMahon, L. Yu, and M. D. Ediger, J. Phys. Chem. Lett. 2, 2683 (2011).

${ }^{31}$ S. S. Dalal, D. M. Walters, I. Lyubimov, J. J. de Pablo, and M. D. Ediger, Proc. Natl. Acad. Sci. U. S. A. 112, 4227 (2015).

${ }^{32}$ K. L. Kearns, H.-Y. Na, R. D. Froese, S. Mukhopadhyay, H. Woodward, D. Welsh, T. De Vries, D. Devore, P. Trefonas, and L. Hong, Proc. SPIE 9183, 91830F (2014).

${ }^{33}$ S. Singh and J. J. de Pablo, J. Chem. Phys. 134, 194903 (2011).

${ }^{34}$ Z. Shi, P. G. Debenedetti, and F. H. Stillinger, J. Chem. Phys. 134, 114524 (2011).

${ }^{35}$ J. A. Torres, P. F. Nealey, and J. J. de Pablo, Phys. Rev. Lett. 85, 3221 (2000).

${ }^{36}$ Z. Yang, Y. Fujii, F. K. Lee, C.-H. Lam, and O. K. C. Tsui, Science 328, 1676 (2010).

${ }^{37}$ D. Qi, M. Ilton, and J. Forrest, Eur. Phys. J. E 34, 1 (2011).

${ }^{38}$ M. D. Ediger and J. A. Forrest, Macromolecules 47, 471 (2014).

${ }^{39}$ L. Zhu, C. Brian, S. Swallen, P. Straus, M. Ediger, and L. Yu, Phys. Rev. Lett. 106, 256103 (2011).

${ }^{40}$ R. Malshe, M. D. Ediger, L. Yu, and J. J. de Pablo, J. Chem. Phys. 134, 194704 (2011)

${ }^{41}$ C. W. Brian and L. Yu, J. Phys. Chem. A 117, 13303 (2013).

${ }^{42}$ W. Zhang, C. Brian, and L. Yu, J. Phys. Chem. B 119, 5071 (2015).

${ }^{43}$ J. D. Stevenson and P. G. Wolynes, J. Chem. Phys. 129, 234514 (2008).

${ }^{44}$ S. Capaccioli, K. L. Ngai, M. Paluch, and D. Prevosto, Phys. Rev. E 86, 051503 (2012).

${ }^{45}$ K. J. Dawson, K. L. Kearns, L. Yu, W. Steffen, and M. D. Ediger, Proc. Natl. Acad. Sci. U. S. A. 106, 15165 (2009).

${ }^{46}$ K. J. Dawson, L. Zhu, L. Yu, and M. D. Ediger, J. Phys. Chem. B 115, 455 (2011).

${ }^{47}$ K. Dawson, L. A. Kopff, L. Zhu, R. J. McMahon, L. Yu, R. Richert, and M. D. Ediger, J. Chem. Phys. 136, 094505 (2012).

${ }^{48}$ A. Gujral, K. A. O'Hara, M. F. Toney, M. L. Chabinyc, and M. Ediger, Chem. Mater. 27, 3341 (2015).

${ }^{49}$ S. S. Dalal and M. D. Ediger, J. Phys. Chem. Lett. 3, 1229 (2012).

${ }^{50}$ D. Yokoyama, A. Sakaguchi, M. Suzuki, and C. Adachi, Org. Electron. 10, 127 (2009).

${ }^{51}$ D. Yokoyama, Y. Setoguchi, A. Sakaguchi, M. Suzuki, and C. Adachi, Adv. Funct. Mater. 20, 386 (2010).

${ }^{52}$ D. Yokoyama, J. Mater. Chem. 21, 19187 (2011).

${ }^{53}$ M. Oh-e, H. Ogata, Y. Fujita, and M. Koden, Appl. Phys. Lett. 102, 101905 (2013).

${ }^{54}$ C. Mayr, M. Taneda, C. Adachi, and W. Brütting, Org. Electron. 15, 3031 (2014).

${ }^{55}$ Y. Shirota, J. Mater. Chem. 15, 75 (2005).

${ }^{56} \mathrm{C}$. Mayr and W. Brütting, Chem. Mater. 27, 2759 (2015).

${ }^{57}$ T. Komino, H. Tanaka, and C. Adachi, Chem. Mater. 26, 3665 (2014).

${ }^{58}$ A. Haji-Akbari and P. G. Debenedetti, J. Chem. Phys. 141, 024506 (2014).

${ }^{59}$ S. Plimpton, J. Comput. Phys. 117, 1 (1995).

${ }^{60}$ W. L. Jorgensen, D. S. Maxwell, and J. Tirado-Rives, J. Am. Chem. Soc. 118, 11225 (1996)

${ }^{61}$ G. A. Kaminski, R. A. Friesner, J. Tirado-Rives, and W. L. Jorgensen, J. Phys. Chem. B 105, 6474 (2001).

${ }^{62}$ U. Essmann, L. Perera, M. L. Berkowitz, T. Darden, H. Lee, and L. G. Pedersen, J. Chem. Phys. 103, 8577 (1995).

${ }^{63}$ B. Hess, C. Kutzner, D. van der Spoel, and E. Lindahl, J. Chem. Theory Comput. 4, 435 (2008).

${ }^{64}$ S. Pronk, S. Páll, R. Schulz, P. Larsson, P. Bjelkmar, R. Apostolov, M. R. Shirts, J. C. Smith, P. M. Kasson, D. van der Spoel, B. Hess, and E. Lindahl, Bioinformatics 29, 845 (2013).

${ }^{65}$ G. Bussi, D. Donadio, and M. Parrinello, J. Chem. Phys. 126, 014101 (2007).

${ }^{66}$ F. Starr, S. Sastry, J. Douglas, and S. Glotzer, Phys. Rev. Lett. 89, 125501 (2002).

${ }^{67}$ D. Yokoyama and C. Adachi, J. Appl. Phys. 107, 123512 (2010).

${ }^{68}$ D. Yokoyama, K.-i. Nakayama, T. Otani, and J. Kido, Adv. Mater. 24, 6368 (2012).

${ }^{69}$ R. M. Lynden-Bell, J. Kohanoff, and M. G. Del Popolo, Faraday Discuss. 129, 57 (2005).

${ }^{70}$ T. Yan, S. Li, W. Jiang, X. Gao, B. Xiang, and G. A. Voth, J. Phys. Chem. B 110, 1800 (2006). 